\title{
Less efficient pattern separation may contribute to age-related spatial memory deficits
}

\author{
Heather M. Holden ${ }^{1}$ and Paul E. Gilbert ${ }^{1,2 *}$ \\ Department of Psychology, San Diego State University, San Diego, CA, USA \\ 2 San Diego State University, University of California San Diego Joint Doctoral Program in Clinical Psychology, San Diego, CA, USA
}

\author{
Edited by: \\ Emma R. Wood, University of \\ Edinburgh, UK \\ Reviewed by: \\ Junming Wang, University of \\ Mississippi Medical Center, USA \\ Ashok Kumar, University of Florida, \\ USA \\ *Correspondence: \\ Paul E. Gilbert, San Diego State \\ University, University of California \\ San Diego Joint Doctoral Program in \\ Clinical Psychology, 6363 Alvarado \\ Court, Suite 103, San Diego, \\ CA 92120, USA. \\ e-mail: pgilbert@sciences.sdsu.edu
}

\begin{abstract}
Spatial memory deficits have been well-documented in older adults and may serve as an early indicator of mild cognitive impairment (MCl) or Alzheimer's disease (AD) in some individuals. Pattern separation is a critical mechanism for reducing potential interference among similar memory representations to enhance memory accuracy. A small but growing literature indicates that spatial pattern separation may become less efficient as a result of normal aging, possibly due to age-related changes in subregions of the hippocampus. This decreased efficiency in spatial pattern separation may be a critical processing deficit that could be a contributing factor to spatial memory deficits and episodic memory impairment associated with aging. The present paper will review recently published studies in humans, non-human primates, and rodents that have examined age-related changes in spatial pattern separation. The potential basic science, translational, and clinical implications from these studies are discussed to illustrate the need for future research to further examine the relationship between spatial pattern separation and brain changes associated with aging and neurodegenerative disease.
\end{abstract}

Keywords: hippocampus, dentate gyrus, aging, spatial memory, interference, pattern separation

\section{SPATIAL MEMORY DECLINE IN AGING}

In the United States, Alzheimer's disease $(\mathrm{AD})$ is the most common cause of dementia in older adults and accounts for $60-80 \%$ of dementia cases (Alzheimer's Association, 2012). In the year 2012, an estimated 5.4 million Americans will be diagnosed with $\mathrm{AD}$, however, this number is projected to increase to 11-16 million by 2050 (Alzheimer's Association, 2012). As a result of the aging "baby boom" generation and increasing longevity in the US population, the disease is a growing public health concern with costs estimated to reach $\$ 200$ billion in 2012 . Although a number of risk factors for $\mathrm{AD}$ have been discussed (e.g., diagnosis of mild cognitive impairment (MCI), family history of $\mathrm{AD}$, apolipoprotein epsilon E4 allele), one of the most well-documented risk factors for the disease is increasing age (Kamboh, 2004). Therefore, a major aim of recent research has focused on identifying early indicators of cognitive dysfunction in older adults.

A variety of cognitive functions may be affected in older adults, however, one of the most commonly reported deficits associated with aging is memory loss. Although not all memory domains are equally affected by aging (e.g., source vs. item memory), some domains such as spatial memory appear to be particularly sensitive to age-related change. Age-related spatial memory decline has been well-documented in both humans (reviewed by Iachini et al., 2009) and animal models (reviewed by Sharma et al., 2010). Spatial memory deficits also have been well-documented in older adults diagnosed with AD (Sahakian et al., 1988; Kessels et al., 2005) or mild cognitive impairment (MCI: Alescio-Lautier et al., 2007; deIpolyi et al., 2007; Hort et al., 2007). Some evidence suggests that spatial memory deficits even may serve as an early indicator of AD (Buccione et al., 2007; deIpolyi et al., 2007; Hort et al., 2007). Age-related spatial memory deficits may result from changes in a variety of brain regions including the hippocampus, temporal lobes, and the frontal-parietal network (Iachini et al., 2009). In particular, spatial memory decline associated with aging has been suggested to result from age-related changes in the hippocampus across species (reviewed by Barnes, 1988). However, it is only recently that studies have begun to examine how agerelated changes in particular subregions of the hippocampus may affect specific mnemonic processes. One such process is spatial pattern separation, a mechanism that may be essential for the accurate formation and retrieval of spatial memories. Less efficient spatial pattern separation in older humans and animals may contribute to impairments in spatial memory, particularly in situations when spatial interference is high.

\section{PATTERN SEPARATION}

Pattern separation is a mechanism for separating partially overlapping patterns of activation so that one pattern may be retrieved as separate from other similar patterns. A pattern separation mechanism may be critical for reducing potential interference among similar memory representations to enhance memory accuracy (Gilbert and Brushfield, 2009). A number of models have suggested that the hippocampus supports pattern separation (Marr, 1971; McNaughton and Nadel, 1990; O'Reilly and McClelland, 1994; Shapiro and Olton, 1994; Rolls and Kesner, 2006; Kesner, 2007; Myers and Scharfman, 2009; Rolls, 2010). In particular, the dentate gyrus (DG) and CA3 subregions of the hippocampus have been shown to play a critical role in 
pattern separation in animal models using electrophysiological methods (McNaughton et al., 1989; Tanila, 1999; Leutgeb et al., 2007), neurotoxin-induced lesions (Gilbert et al., 2001; Lee et al., 2005; Gilbert and Kesner, 2006; Goodrich-Hunsaker et al., 2008; McTighe et al., 2009; Morris et al., 2012), and genetic manipulations (Kubik et al., 2007; McHugh et al., 2007). In addition, studies using high-resolution functional magnetic resonance imaging (fMRI) have shown that the human hippocampus (Kirwan and Stark, 2007), and specifically the DG/CA3 subregions (Bakker et al., 2008; Lacy et al., 2011), are active during pattern separation tasks (also see reviews by Carr et al., 2010; Yassa and Stark, 2011b).

\section{AGE-RELATED CHANGES IN THE BRAIN}

Aging has been shown to result in gray matter and white matter changes in a variety of brain regions (Allen et al., 2005; Ziegler et al., 2008; Driscoll et al., 2009; Kennedy and Raz, 2009), including the hippocampus (Good et al., 2001; Allen et al., 2005; Driscoll and Sutherland, 2005; Raz et al., 2005; Walhovd et al., 2010). Longitudinal studies have reported that non-demented older adults show decreased volume in the hippocampus and parahippocampal cortices (Driscoll et al., 2009). However, hippocampal volume has been reported to decrease at a faster rate than other medial temporal lobe structures (Raz et al., 2004). Longitudinal changes in hippocampal volume have been shown to be the primary determinant of memory decline (Mungas et al., 2005; Kramer et al., 2007). fMRI signal intensity has been shown to decline in all hippocampal subregions in older adults (Small et al., 2002). However, the DG subregion may be particularly susceptible to age-related changes in both humans (Small et al., 2002) and animal models (Small et al., 2004; Patrylo and Williamson, 2007). In addition, significant age-related changes have been documented in the perforant pathway in both humans (Yassa et al., 2011a) and animal models (Geinisman et al., 1992). This diminished input from the entorhinal cortex to the DG also may impact connections to the CA3 subregion and this reduction in connectivity was shown to reliably predict spatial learning deficits in old rats (Smith et al., 2000).

\section{PATTERN SEPARATION AND AGING}

There is a small but growing literature suggesting that pattern separation may be adversely affected by aging. Wilson et al. (2006) proposed a model to account for age-related susceptibility to interference. This model suggests that age-related changes in the DG subregion of the hippocampus may result in less efficient pattern separation due to an impaired ability to reduce similarity among new input patterns. Furthermore, age-related changes in the hippocampus strengthen the autoassociative network of the CA3 subregion that supports pattern completion, a mechanism that allows a complete representation of stored information to be retrieved using partial cues. These age-related changes may cause the CA3 subregion of the hippocampus to become entrenched in pattern completion at the expense of processing new information and pattern separation. As further evidence for this hypothesis, a recent study by Yassa et al. (2011a) reported that reduced pattern separation activity in the DG/CA3 regions of aged humans was linked to structural changes in the perforant pathway. The changes were suggested to weaken the processing of novel information while strengthening the processing of stored information. Shing et al. (2011) report that increased false alarm rates in older adults when performing an episodic memory task may be linked to CA3-4/DG volume, which may reflect individual agerelated differences in maintaining a pattern separation-pattern completion equilibrium.

Given the well-documented role of the DG in supporting pattern separation and the potential susceptibility of this region to age-related change, recent behavioral studies have begun to examine pattern separation in older humans. Using a continuous recognition paradigm developed by Kirwan and Stark (2007), Toner et al. (2009) reported that non-demented older adults are impaired on a pattern separation task for visual object information. A subsequent fMRI study supported these findings and additionally demonstrated increased age-related activity in the DG and CA3 subregions when pattern separation demands were high on a visual object task (Yassa et al., 2010a).

\section{SPATIAL PATTERN SEPARATION IN OLDER HUMANS}

Recent studies also have begun to examine spatial pattern separation deficits in older adults. A study by Stark et al. (2010) reported that spatial pattern separation is impaired in a subset of older adults. Participants studied unique pairs of pictures and later were asked to identify whether the pictures were both in the same location as before or whether one of the pictures was in a different location. In the same condition, neither of the pictures in a pair was moved. In the three different conditions (close, medium, far) the location of one of the pictures in a pair was moved by varying both the distance and the angle from the original location. Older adults were separated into an aged impaired group and aged unimpaired group, based on standardized word learning task performance. Analyses revealed performance deficits on the close, medium, and far trials for the aged impaired group relative to both the aged unimpaired and young groups. Therefore, the findings suggest that spatial pattern separation may be impaired in a subset of older adults.

A subsequent study by Holden et al. (2012) examined the ability of cognitively normal young and older adults to perform a spatial memory task involving varying degrees of spatial interference to assess spatial pattern separation. During the sample phase of each trial, a circle appeared briefly on a computer screen. The participant was instructed to remember the location of the circle. During the choice phase, two circles were displayed simultaneously and the participant was asked to indicate which circle was in the same location as the sample phase circle. The two choice phase circles were separated by one of four possible spatial separations across trials: $0,0.5,1.0$, and $1.5 \mathrm{~cm}$. Smaller separations are likely to create increased overlap among memory representations, which may result in heightened interference and a greater need for pattern separation. Consistent with this hypothesis, the performance of both young and older adults increased as a function of increased spatial separation. However, young adults outperformed older adults, suggesting that spatial pattern separation may be less efficient in older adults. In an attempt to replicate the findings of Stark et al. (2010), the older adult group was separated into older impaired and older unimpaired groups based 
on performance on a standardized delayed word recall measure. Consistent with Stark et al. (2010), the older impaired group was significantly impaired relative to both the older unimpaired and young groups.

\section{SPATIAL PATTERN SEPARATION IN OLDER ANIMALS}

Kubo-Kawai and Kawai (2007) conducted a study in aged monkeys that is similar in nature to the two aforementioned studies conducted in older humans. This study tested young and older monkeys on a delay non-match-to-position task involving manipulations of the spatial distance between two locations. During the sample phase, a plate was positioned to cover a baited food well along a single row of four food wells. The animal displaced the plate to receive a reward. During the choice phase, an identical plate was positioned to cover the unbaited sample phase well (incorrect choice) and a second identical plate was positioned to cover a different baited food well in one of the three remaining locations (correct choice). The distance between the two choice phase plates was manipulated across trials and included separations of 12,24 , and $36 \mathrm{~cm}$. The data showed that the ability of both young and older monkeys to choose the correct plate improved as a function of increased spatial separation between the two plates during the choice phase. However, no age-related differences in performance were detected across separations. In a second experiment, the sample phase followed the same procedure described for the previous experiment. However, on the choice phase, two rows of food wells were used. The plate covering the unbaited sample phase food well (incorrect choice) was located on one row, whereas the plate covering the new location was positioned to cover a baited food well (correct choice) on a different row of wells. Spatial separations of 15, 25.6, and $37.1 \mathrm{~cm}$ were used to separate the plates across choice phase trials. The data revealed that older monkeys were impaired relative to young monkeys across all three separations, with the largest group differences found on trials involving the closest $15 \mathrm{~cm}$ separation. One could speculate that smaller separations were likely to create increased overlap among memory representations, which may result in heightened interference and a greater need for pattern separation. Although this study was not designed or interpreted by the authors to assess pattern separation, these findings in aged monkeys are in accordance with the findings from the studies involving older humans.

A recent study from our lab (Gracian et al., unpublished observations) provides further behavioral evidence that spatial pattern separation may be impaired in aged animals. Young and old rats were trained on a radial eight-arm maze to discriminate between a rewarded arm and a non-rewarded arm that were either adjacent to one another (high spatial interference) or separated by a distance of two arm positions (low spatial interference). Performance on this task recently was shown to be dependent on the DG hippocampal subregion (Morris et al., 2012). Gracian et al. showed that young and old rats committed similar numbers of errors in the separated condition. However, in the adjacent condition, old rats committed significantly more errors compared to young rats. The results suggest that decreased spatial pattern separation in aged rats may impair performance on the adjacent condition, which involved greater spatial interference among distal cues. However, performance increased in the separated condition when there was less overlap among distal cues and less need for pattern separation.

A recently published study by Marrone et al. (2011) provides further neurobiological insight into how age-related changes in the DG may affect pattern separation and spatial memory. In this study, young and aged rats were allowed to explore either: (1) the same environment on two occasions or (2) one environment followed by a different environment. A marker of cellular activity (zif268/egr1) was used to examine granule cell activity during exploration of the environments. Older animals were found to recruit distinct populations of granule cells during exploration of the same environment on two different occasions, which the authors interpret to be an indication of greater pattern separation. However, when the aged animals visited different environments, the reliability of repeated zif268 expression in young and aged animals was comparable, thus the age-related increase in pattern separation was no longer apparent. The study also found that increased pattern separation in the similar contexts was correlated with a reduced ability of older animals to disambiguate similar contexts during a sequential spatial recognition task. The authors conclude that spatial memory performance in aged animals is most impaired in situations where interference is increased, presumably due to decreased pattern separation. Collectively, the aforementioned studies offer evidence that spatial pattern separation may become less efficient as a result of aging.

\section{IMPLICATIONS}

Age-related memory decline has been suggested to stem from subregion-specific epigenetic and transcriptional changes in the hippocampus (Penner et al., 2010). For example, neurogenesis is reduced in aged animals (Kuhn et al., 1996) and is related to decreased hippocampal volume and impaired performance on hippocampal-dependent tasks (Driscoll et al., 2006). These newborn neurons may be involved in mnemonic processes particularly dependent on the DG subregion, such as pattern separation (Clelland et al., 2009; Aimone et al., 2010, 2011; Creer et al., 2010; Deng et al., 2010; Sahay et al., 2011), whereas older DG cells may contribute to pattern completion (Nakashiba et al., 2012). Sahay et al. (2011) suggest that interventions that increase neurogenesis during adulthood may have clinical implications for reversing age-related impairments in pattern separation and associated DG dysfunction. Therefore, the development of behavioral tasks sensitive to age-related changes in spatial pattern separation may have implications for future studies of neurogenesis.

Age-related changes in spatial pattern separation also may contribute to episodic memory deficits, which have been welldocumented in older adults (Rand-Giovannetti et al., 2006) and are a prominent feature of $\mathrm{AD}$ that may be detectable several years before disease onset (Bondi et al., 1999). Episodic memory also is impaired in non-demented older adults who are at risk for AD based on genetics (Saunders et al., 1993) or a diagnosis of MCI (Hodges et al., 2006). One key feature of episodic memory that differentiates it from other types of memory is that the elements of an episodic memory must be associated into a context to demarcate the episode in space and time. In addition, a pattern separation mechanism may be necessary to separate 
the elements of different episodic memories to avoid interference (Gilbert et al., 2001). It is possible that less efficient spatial pattern separation in older adults may result in poorer memory due to increased interference among the spatial components of episodic memories. The identification of a key mnemonic processing deficit in pattern separation may result in behavioral interventions that structure daily living tasks to mitigate interference in the spatial domain and potentially improve spatial and episodic memory in older adults.

Normal and pathological aging may have differential effects on hippocampal subregions. The DG subregion may be particularly susceptible to age-related changes in humans, however, there may be less impact on pyramidal cells in the CA subregions (Small et al., 2002). In contrast, the CA subregions may be more vulnerable to pathological changes associated with AD (Braak and Braak, 1996; West et al., 2000; Price et al., 2001; Apostolova et al., 2010). As mentioned previously, a primary goal in AD research is to identify risk factors and preclinical markers of the disease in older adults. Given the differential effects of normal aging and AD on the various subregions of the hippocampus, tasks that are sensitive to dysfunction in particular subregions, such as measures of pattern separation, may help to differentiate between cognitive impairment associated with normal aging and pathological changes associated with $\mathrm{AD}$. In support of this idea, a recent study by Yassa et al. (2010b) reported that individuals with amnestic MCI show impairments compared to healthy older adults on an

\section{REFERENCES}

Aimone, J. B., Deng, W., and Gage, F. H. (2010). Adult neurogenesis: integrating theories and separating functions. Trends Cogn. Sci. 14, 325-337.

Aimone, J. B., Deng, W., and Gage, F. H. (2011). Resolving new memories: a critical look at the dentate gyrus, adult neurogenesis, and pattern separation. Neuron 70, 589-596.

Alescio-Lautier, B., Michel, B. F., Herrera, C., Elahmadi, A., Chambon, C., Touzet, C., and Paban, V. (2007). Visual and visuospatial short-term memory in mild cognitive impairment and Alzheimer disease: role of attention. Neuropsychologia 45, 1948-1960.

Allen, J. S., Bruss, J., Brown, C. K., and Damasio, H. (2005). Normal neuroanatomical variation due to age: the major lobes and a parcellation of the temporal region. Neurobiol. Aging 26, 1245-1260.

Alzheimer's Association. (2012). Alzheimer's Association report: 2012 Alzheimer's disease facts and figures. Alzheimers Dement. 8, 131-168.

Apostolova, L. G., Hwang, K. S., Andrawis, J. P., Green, A. E., Babakchanian, S., Morra, J. H., Cummings, J. L., Toga, A. W., Trojanowski, J. Q., Shaw, L. M.,

object recognition memory task that taxes pattern separation. In addition, structural and functional changes were observed in the DG/CA3 hippocampal subregions of these individuals.

\section{CONCLUSIONS}

In conclusion, spatial memory deficits have been welldocumented in older adults and may serve as an early indicator of MCI or AD in some individuals. The operation of a pattern separation mechanism may be critical for reducing interference among similar memory representations to enhance memory accuracy. Evidence suggests that brain regions critical to pattern separation, including the DG and CA3 hippocampal subregions, may be particularly susceptible to adverse age-related changes. A small but growing literature indicates that spatial pattern separation may become less efficient as a result of normal aging. This decreased efficiency in pattern separation may contribute to spatial memory deficits and episodic memory impairment associated with aging. Based on the aforementioned studies, it is clear that additional research is needed to examine the relationship between spatial pattern separation and brain changes associated with aging and neurodegenerative disease.

\section{ACKNOWLEDGMENTS}

A portion of the research reviewed in the paper was supported by a National Institutes of Health Grant (\#AG034202) from the National Institute on Aging awarded to Paul E. Gilbert.

Alzheimer's disease. Eur. J. Neurol. 14, 440-446.

Carr, V. A., Rissman, J., and Wagner, A. D. (2010). Imaging the human medial temporal lobe with highresolution fMRI. Neuron 65, 298-308.

Clelland, C. D., Choi, M., Romberg, C., Clemenson, G. D. Jr., Fragniere, A., Tyers, P., Jessberger, S., Saksida, L. M., Barker, R. A., Gage, F. H., and Bussey, T. J. (2009). A functional role for adult hippocampal neurogenesis in spatial pattern separation. Science 325, 210-213.

Creer, D. J., Romberg, C., Saksida, L. M., van Praag, H., and Bussey, T. J. (2010). Running enhances spatial pattern separation in mice. Proc. Natl. Acad. Sci. U.S.A. 107, 2367-2372.

deIpolyi, A. R., Rankin, K. P., Mucke, L., Miller, B. L., and Gorno-Tempini, M. L. (2007). Spatial cognition and the human navigation network in AD and MCI. Neurology 69, 986-997.

Deng, W., Aimone, J. B., and Gage, F. H. (2010). New neurons and new memories: how does adult hippocampal neurogenesis affect learning and memory? Nat. Rev. Neurosci. 11, 339-350.

Driscoll, I., Davatzikos, C., An, Y., Wu, X., Shen, D., Kraut, M., and Resnick,
S. M. (2009). Longitudinal pattern of regional brain volume change differentiates normal aging from MCI. Neurology 72, 1906-1913.

Driscoll, I., Howard, S. R., Monfils, M. H., Tomanek, B., Brooks, W. M., and Sutherland, R. J. (2006). The aging hippocampus: a multi-level analysis in the rat. Neuroscience 139, 1173-1185.

Driscoll, I., and Sutherland, R. J. (2005). The aging hippocampus: navigating between rat and human experiments. Rev. Neurosci. 16, 87-121.

Geinisman, Y., deToledo-Morrell, L., Morrell, F., Persina, I. S., and Rossi, M. (1992). Age-related loss of axospinous synapses formed by two afferent systems in the rat dentate gyrus as revealed by the unbiased stereological dissector technique. Hippocampus 2, 437-444.

Gilbert, P. E., Kesner, R. P., and Lee, I. (2001). Dissociating hippocampal subregions: double dissociation between dentate gyrus and CA1. Hippocampus 11, 626-636.

Gilbert, P. E., and Brushfield, A. M. (2009). The role of the CA3 hippocampal subregion in spatial memory: a process oriented behavioral assessment. Prog. Neuropsychopharmacol. Biol. Psychiatry 33, 774-781. 
Gilbert, P. E., and Kesner, R. P. (2006). The role of the dorsal CA3 hippocampal subregion in spatial working memory and pattern separation. Behav. Brain Res. 169, 142-149.

Good, C. D., Johnsrude, I. S., Ashburner, J., Henson, R. N., Friston, K. J., and Frackowiak, R. S. (2001). A voxel-based morphometric study of ageing in 465 normal adult human brains. Neuroimage 14, 21-36.

Goodrich-Hunsaker, N. J., Hunsaker, M. R., and Kesner, R. P. (2008). The interactions and dissociations of the dorsal hippocampus subregions: how the dentate gyrus, CA3, and CA1 process spatial information. Behav. Neurosci. 122, 16-26.

Hodges, J. R., Erzinclioglu, S., and Patterson, K. (2006). Evolution of cognitive deficits and conversion to dementia in patients with mild cognitive impairment: a very-long-term follow-up study. Dement. Geriatr. Cogn. Disord. 21, 380-391.

Holden, H. M., Hoebel, C., Loftis, K., and Gilbert, P. E. (2012). Spatial pattern separation in cognitively normal young and older adults. Hippocampus. doi: 10.1002/hipo.22017. [Epub ahead of print].

Hort, J., Laczó, J., Vyhnálek, M., Bojar, M., Bures, J., and Vlcek, K. (2007). Spatial navigation deficit in amnestic mild cognitive impairment. Proc. Natl. Acad. Sci. U.S.A. 104, 4042-4047.

Iachini, I., Iavarone, A., Senese, V. P., Ruotolo, F., and Ruggiero, G. (2009). Visuospatial memory in healthy elderly, AD and MCI: a review. Curr. Aging Sci. 2, 43-59.

Kamboh, M. I. (2004). Molecular genetics of late-onset Alzheimer's disease. Ann. Hum. Genet. 68, 381-404.

Kennedy, K. M., and Raz, N. (2009). Aging white matter and cognition: differential effects of regional variations in diffusion properties on memory, executive functions, and speed. Neuropsychologia 47, 916-927.

Kesner, R. P. (2007). A behavioral analysis of dentate gyrus function. Prog. Brain Res. 163, 567-576.

Kessels, R. P., Boekhorst, S. T., and Postma, A. (2005). The contribution of implicit and explicit memory to the effects of errorless learning: a comparison between young and older adults. J. Int. Neuropsychol. Soc. 11, 144-151.

Kirwan, C. B., and Stark, C. E. (2007). Overcoming interference: an fMRI investigation of pattern separation in the medial temporal lobe. Learn. Mem. 14, 625-633.

Kramer, J. H., Mungas, D., Reed, B. R., Wetzel, M. E., Burnett, M. M., Miller, B. L., Weiner, M. W., and Chui, H. C. (2007). Longitudinal MRI and cognitive change in healthy elderly. Neuropsychology 21 , 412-418.

Kubik, S., Miyashita, T., and Guzowski, J. F. (2007). Using immediate-early genes to map hippocampal subregional functions. Learn. Mem. 14, 758-770.

Kubo-Kawai, N., and Kawai, N. (2007). Interference effects by spatial proximity and age-related declines in spatial memory by Japanese monkeys (Macaca fuscata): deficits in the combined use of multiple spatial cues. J. Comp. Psychol. 121, 189-197.

Kuhn, H. G., Dickinson-Anson, H., and Gage, F. H. (1996). Neurogenesis in the dentate gyrus of the adult rat: age-related decrease of neuronal progenitor proliferation. J. Neurosci. 16, 2027-2033.

Lacy, J. W., Yassa, M. A., Stark, S. M., Muftuler, L. T., and Stark, C. E. (2011). Distinct pattern separation related transfer functions in human CA3/dentate and CA1 revealed using high-resolution $\mathrm{fMRI}$ and variable mnemonic similarity. Learn. Mem. 18, 15-18.

Lee, I., Jerman, T. S., and Kesner, R P. (2005). Disruption of delayed memory for a sequence of spatial locations following CA1- or CA3lesions of the dorsal hippocampus. Neurobiol. Learn. Mem. 84 138-147.

Leutgeb, J. K., Leutgeb, S., Moser, M. B., and Moser, E. I. (2007). Pattern separation in the dentate gyrus and CA3 of the hippocampus. Science 315, 961-966.

Marr, D. (1971). Simple memory: a theory for archicortex. Proc. R. Soc. Lond. B Biol. Sci. 262, 23-81.

Marrone, D. F., Adams, A. A., and Satvat, E. (2011). Increased pattern separation in the aged fascia dentata. Neurobiol Aging 32, 2317.e23-e32.

McHugh, T. J., Jones, M. W., Quinn, J. J., Balthasar, N., Coppari, R., Elmquist, J. K., Lowell, B. B. Fanselow, M. S., Wilson, M. A., and Tonegawa, S. (2007). Dentate gyrus NMDA receptors mediate rapid pattern separation in the hippocampal network. Science 317, 94-99.

McNaughton, B. L., Barnes, C. A. Meltzer, J., and Sutherland, R. J. (1989). Hippocampal granule cells are necessary for normal spatial learning but not for spatially-selective pyramidal cell discharge. Exp. Brain Res. 76, 485-496.

McNaughton, B. L., and Nadel, L. (1990). "Hebb-Marr networks and the neurobiological representation of action in space," in Neuroscience and Connectionist Theory, eds $\mathrm{M}$. A. Gluck and D. E. Rumelhart (Hillsdale, NJ: Erlbaum), 1-63.

McTighe, S. M., Mar, A. C., Romberg, C., Bussey, T. J., and Saksida, L. M. (2009). A new touchscreen test of pattern separation: effect of hippocampal lesions. Neuroreport 20 , 881-885.

Morris, A. M., Churchwell, J. C. Kesner, R. P., and Gilbert, P. E (2012). Selective lesions of the dentate gyrus produce disruptions in place learning for adjacent spatial locations. Neurobiol. Learn. Mem. 97, 326-331.

Mungas, D., Harvey, D., Reed, B. R. Jagust, W. J., DeCarli, C., Beckett, L., Mack, W. J., Kramer, J. H., Weiner, M. W., Schuff, N., and Chui, H. C. (2005). Longitudinal volumetric MRI change and rate of cognitive decline. Neurology 65, 565-571.

Myers, C. E., and Scharfman, H. E. (2009). A role for hilar cells in pattern separation in the dentate gyrus: a computational approach. Hippocampus 19, 321-337.

Nakashiba, T., Cushman, J. D., Pelkey, K. A., Renaudineau, S., Buhl, D. L., McHugh, T. J., Barrera, V. R. Chittajallu, R., Iwamoto, K. S. McBain, C. J., Fanselow, M. S., and Tonegawa, S. (2012). Young dentate granule cells mediate pattern separation, whereas old granule cells facilitate pattern completion. Cell 149, 188-201.

O'Reilly, R. C., and McClelland, J. L. (1994). Hippocampal conjunctive encoding, storage, and recall: avoiding a trade-off. Hippocampus 4, 661-682.

Patrylo, P. R., and Williamson, A. (2007). The effects of aging on dentate circuitry and function. Prog. Brain Res. 163, 679-696.

Penner, M. R., Roth, T. I., Chawla, M. K., Hoang, L. T., Roth, E. D. Lubin, F. D., Sweatt, J. D., Worley, P. F., and Barnes, C. A. (2010). Age related changes in Arc transcription and DNA methylation within the hippocampus. Neurobiol. Aging 32, 2198-2210.

Price, J. L., Ko, A. I., Wade, M. J., Tsou, S. K., McKeel, D. W., and Morris, J. C. (2001). Neuron number in the entorhinal cortex and CAl in preclinical Alzheimer disease. Arch. Neurol. 58, 1395-1402.

Rand-Giovannetti, E., Chua, E. F., Driscoll, A. E., Schacter, D. L.,
Albert, M. S., and Sperling, R. A. (2006). Hippocampal and neocortical activation during repetitive encoding in older persons. Neurobiol. Aging 27, 173-182.

Raz, N., Gunning-Dixon, F., Head, D., Rodrigue, K. M., Williamson, A., and Acker, J. D. (2004). Aging, sexual dimorphism, and hemispheric asymmetry of the cerebral cortex: replicability of regional differences in volume. Neurobiol. Aging 25, 377-396.

Raz, N., Lindenberger, U., Rodrigue, K. M., Kennedy, K. M., Head, D., Williamson, A., Dahle, C., Gerstorf, D., and Acker, J. D. (2005). Regional brain changes in aging healthy adults: general trends, individual differences and modifiers. Cereb. Cortex 15, 1676-1689.

Rolls, E. T. (2010). A computational theory of episodic memory formation in the hippocampus. Behav. Brain Res. 215, 180-196.

Rolls, E. T., and Kesner, R. P. (2006). A computational theory of hippocampal function, and empirical tests of the theory. Prog. Neurobiol. 79, $1-48$.

Sahakian, B. J., Morris, R. G., Evenden, J. L., Heald, A., Levy, R., Philpot, M., and Robbins, T. W. (1988). A comparative study of visuospatial memory and learning in Alzheimer-type dementia and Parkinson's disease. Brain 111, 695-718.

Sahay, A., Wilson, D. A., and Hen, R. (2011). Pattern separation: a common function for new neurons in hippocampus and olfactory bulb. Neuron 70, 582-588.

Saunders, A. M., Strittmatter, W. J., Schmechel, D., George-Hyslop, P. H., Pericak-Vance, M. A., Joo, S. H., Rosi, B. L., Gusella, J. F., CrapperMacLachlan, D. R., Alberts, M. J., Hulette, C., Crain, B., Goldgaber, D. and Roses, A. D. (1993). Association of apolipoprotein E alleles epsilon 4 with late-onset familial and sporadic Alzheimer's disease. Neurology 43, 1467-1472.

Shapiro, M. L., and Olton, D. S. (1994). "Hippocampal Function and Interference," in Memory Systems, eds D. L. Schacter and E. Tulving (London: MIT), 141-146.

Sharma, S., Rakoczy, S., and BrownBorg, H. (2010). Assessment of spatial memory in mice. Life Sci. 87 521-536.

Shing, Y. L., Rodrigue, K. M., Kennedy, K. M., Fandakova, Y., Bodammer, N., Werkle-Bergner, M., Lindenberger, U., and Raz, N. (2011). Hippocampal subfield volumes: age, vascular risk, and 
correlation with associative memory. Front. Aging Neurosci. 3:2. doi: 10.3389/fnagi.2011.00002

Small, S. A., Chawla, M. K., Buonocore, M., Rapp, P. R., and Barnes, C. A. (2004). Imaging correlates of brain function in monkeys and rats isolates a hippocampal subregion differentially vulnerable to aging. Proc. Natl. Acad. Sci. U.S.A. 101, 7181-7186.

Small, S. A., Tsai, W. Y., DeLaPaz, R., Mayeux, R., and Stern, Y. (2002). Imaging hippocampal function across the human life span: is memory decline normal or not? Ann. Neurol. 51, 290-295.

Smith, T. D., Adams, M. M., Gallagher, M., Morrison, J. H., and Rapp, P. R. (2000). Circuit-specific alterations in hippocampal synaptophysin immunoreactivity predict spatial learning impairment in aged rats. J. Neurosci. 20, 6587-6593.

Stark, S. M., Yassa, M. A., and Stark, C. E. (2010). Individual differences in spatial pattern separation performance associated with healthy aging in humans. Learn. Mem. 17, 284-288.
Tanila, H. (1999). Hippocampal place cells can develop distinct representations of two visually identical environments. Hippocampus 9, 235-246.

Toner, C. K., Pirogovsky, E., Kirwan, C. B., and Gilbert, P. E. (2009). Visual object pattern separation deficits in nondemented older adults. Learn. Mem. 16, 338-342.

Walhovd, K. B., Fjell, A. M., Dale, A. M., McEvoy, L. K., Brewer, J., Karow, D. S., Salmon, D. P., and Fennema-Notestine, C., Alzheimer's Disease Neuorimaging Initiative. (2010). Multi-modal imaging predicts memory performances in normal aging and cognitive decline. Neurobiol. Aging 31, 1107-1112.

West, M. J., Kawas, C. H., Martin, L. J., and Troncoso, J. C. (2000). The CA1 region of the human hippocampus is a hot spot in Alzheimer's disease. Ann. N.Y. Acad. Sci. 908, 255-259.

Wilson, I. A., Gallagher, M., Eichenbaum, H., and Tanila, $\mathrm{H}$. (2006). Neurocognitive aging: prior memories hinder new hippocampal encoding. Trends Neurosci. 29, 662-670.

Yassa, M. A., Lacy, J. W., Stark, S. M., Albert, M. S., Gallagher, M., and Stark, C. E. (2010a). Pattern separation deficits associated with increased hippocampal CA3 and dentate gyrus activity in nondemented older adults. Hippocampus 21, 968-979.

Yassa, M. A., Mattfeld, A. T., Stark, S. M., and Stark, C. E. (2011a). Age-related memory deficits linked to circuit-specific disruptions in the hippocampus. Proc. Natl. Acad. Sci. U.S.A. 108, 8873-8878.

Yassa, M. A., Stark, S. M., Bakker, A., Albert, M. S., Gallagher, M., and Stark, C. E. (2010b). Highresolution structural and functional MRI of hippocampal CA3 and dentate gyrus in patients with amnestic Mild Cognitive Impairment. Neuroimage 51, 1242-1252.

Yassa, M. A., and Stark, C. E. (2011b). Pattern separation in the hippocampus. Trends. Neurosci. 34, 515-525.

Ziegler, D. A., Piguet, O., Salat, D. H., Prince, K., Connally, E., and
Corkin, S. (2008). Cognition in healthy aging is related to regional white matter integrity, but not cortical thickness. Neurobiol. Aging 31, 1912-1926.

Conflict of Interest Statement: The authors declare that the research was conducted in the absence of any commercial or financial relationships that could be construed as a potential conflict of interest.

Received: 29 March 2012; paper pending published: 27 April 2012; accepted: 14 May 2012; published online: 29 May 2012.

Citation: Holden HM and Gilbert PE (2012) Less efficient pattern separation may contribute to age-related spatial memory deficits. Front. Ag. Neurosci. 4:9. doi: 10.3389/fnagi.2012.00009

Copyright (c) 2012 Holden and Gilbert. This is an open-access article distributed under the terms of the Creative Commons Attribution Non Commercial License, which permits non-commercial use, distribution, and reproduction in other forums, provided the original authors and source are credited. 